\title{
Challenges in Seniors Adopting Assistive Robots: A Systematic Review
}

\author{
Arni Ariani, Vasvi Kapadia \\ World Health Organization Collaborating Centre on eHealth, University of New South Wales, Australia \\ Email:arniariani@yahoo.com,shah.vasvi@gmail.com
}

\author{
Amir Talaei-Khoei \\ Department of Information Systems, University of Nevada, Reno, United States of America \\ School of Software, University of Technology Sydney, Australia \\ Email: atalaeikhoei@unr.edu
}

Junhua Li

HammondCare, NSW, Australia

Email: junhua.li.syd@gmail.com

Pradeep K. Ray

University of Michigan Joint Institute, Shanghai Jiao Tong University, China

World Health Organization Collaborating Centre on eHealth, University of New South Wales, Australia

Email: pradeep.ray@sjtu.edu.cn

\begin{abstract}
This study aimed to explore challenges underlying seniors' adoption of assistive robots. A systematic literature review involved three stages: the first two stages focused on identifying and selecting relevant papers, followed by extracting and analyzing data from selected papers. Analysis revealed several issues which were categorized based on robot type: rehabilitation or socially assistive. Negative influences related to seniors' concerns about cost, design issues, negative perceptions, safety and privacy concerns; positive influences included ease of use and potential benefits.
\end{abstract}

Keywords: Assistive robot, challenges, seniors, technology adoption.

\section{Introduction}

Australia and other countries worldwide are facing a "greying population". The proportion of Australians aged over 65 years was $13.5 \%$ of 22.5 million people in 2010 , and forecast to reach $22.7 \%$ of 35.9 million people by the year $2050 .{ }^{1}$ The rapid increase in seniors raises many challenges for communities and the Australian healthcare system, including demand for healthcare services for people experiencing deteriorating health, physical and psychological abilities, and the limited number of healthcare staff. ${ }^{2}$

Development in robotic technologies to potentially promote physical and emotional wellbeing of seniors and reduce the workload of healthcare staff has attracted enthusiasm from researchers around the world. ${ }^{2}$ Previously, the term 'assistive robot' was defined as a robot that can help a disabled person in their daily life. ${ }^{3}$ However, that definition does not encompass the range of functionalities of an assistive robot. The term was more recent defined to include two kinds of assistive robots for seniors: rehabilitation and socially assistive robots. $^{4}$

Rehabilitation robots can be used as treatment devices for upper and lower extremity rehabilitation, for example, semiautonomous prostheses. ${ }^{5}$ Robots can be used in a series of rehabilitation therapies to restore functionality of limbs in individuals with stroke, brain trauma or neuromotor disorders. ${ }^{5}$ Furthermore, they can be used as physical and mobility supports for people with non-functioning limbs or after amputation. ${ }^{5}$

Socially assistive robots can be used for social interaction, for example, companionship or service. ${ }^{4}$ The majority of companionship robots are in the form of a pet to enable mutual affection. ${ }^{4}$ These robots can help enhance health and psychological wellbeing of seniors. ${ }^{4}$ Service robots are designed to assist seniors in their daily activities such as eating, housework or medication reminders, potentially helping seniors to remain at home for longer. ${ }^{4}$ For example, when part of a cognitive stimulation program, a robot can act as a cognitive 
prosthesis by reminding the patient to take correct doses of medication on time and to attend appointments. ${ }^{6}$

Despite the fact that assistive robots are being created to support the needs of seniors, adoption of such systems is still in its infancy and remains questionable. ${ }^{2}$ A recent study showed that seniors in aged care facilities refused or resisted to adopt assistive robots, thereby causing robot production to be discontinued. ${ }^{2} \mathrm{~A}$ reasonable explanation for this might be that researchers and developers do not yet fully understand seniors' preferences and needs related to assistive robots. ${ }^{2}$

To date, robotic studies have focused on the development and use of assistive robots for frail and disabled seniors, but there is a paucity of research investigating the challenges and success factors of seniors adopting assistive robots. Gaining more acceptance and adoption by the older population is important for effective use of the assistive robot. According to a report by Gurley et al., ${ }^{7}$ around one third of existing assistive technologies are neglected after being used for one year. In addition, due to time and financial constraints, assistive technology developers do not always conduct market acceptance trials with targeted customers, in this case, seniors. To address this knowledge gap, this systematic review investigates the critical challenges influencing seniors' adoption of assistive robots.

\section{Methodology}

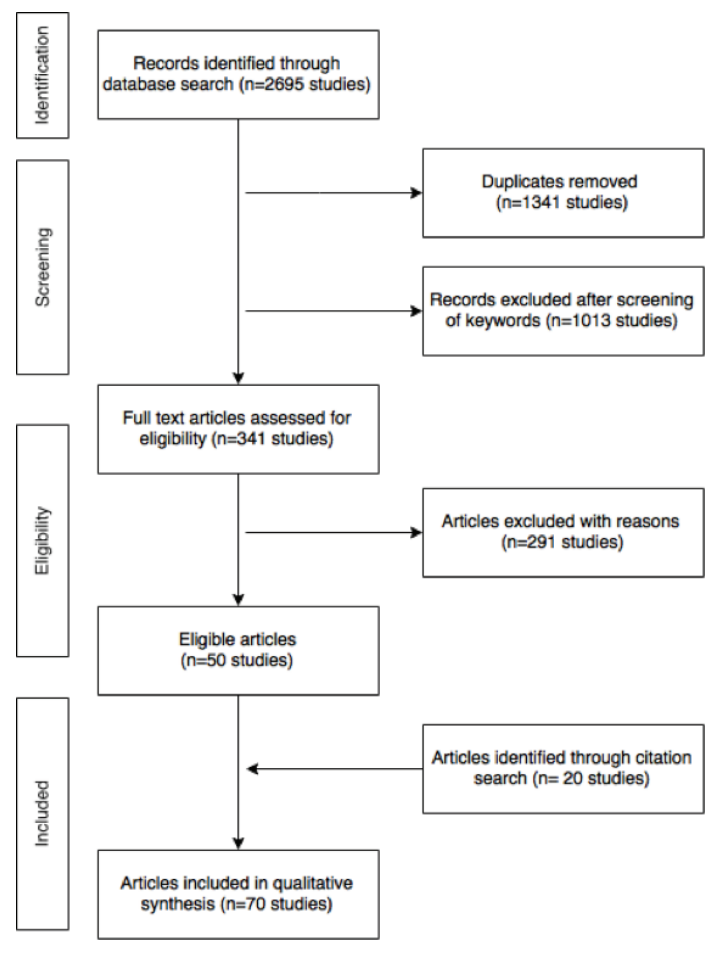

Fig. 1. Identification of resources and search strategy
This systematic review complied with the Preferred Reporting Items for Systematic Reviews and MetaAnalyses (PRISMA) statement and guidelines. ${ }^{8}$ The methodology is summarized in Figure 1.

\subsection{Identification of Resources and Search Strategy}

For this review, we selected scientific papers from two different sources: electronic databases and citation searches of selected papers. A total of nine databases (CINAHL, ERIC, EMBASE, IEEE Xplorer, MEDLINE, Proquest Central, PubMed, PsycINFO, and Web of Science) were selected as being the most frequently used in our research community, providing access to research with in-depth understanding of the current position of assistive robots in an aging society, defined as a large number of citations retrieved from those databases. In order to conduct an extensive and comprehensive search of the literature, we selected the following keywords closely related to the objectives of our research: assistive robots, assistive technology or robotics system; older people, elderly or seniors; and aged care or nursing home.

The search was carried out by using the above identified keywords in combination with Boolean connectors (AND, OR). As the use of assistive robots among seniors is still in its infancy, the literature search was limited to studies published between January 2005 and December 2015. All retrieved papers $(n=2695)$ were stored in JabRef (an open source bibliography reference manager). Duplicates were removed if: the same paper was listed on more than one database (Type I); the same research was published in different venues (e.g. journals, conference proceedings; Type II). Papers with the same titles or the same authors were also removed. At the end of this process, 1354 papers were selected after removing 1341 all duplicate papers (as shown in Figure 1).

\subsection{Selection of Relevant Papers}

In the first stage of screening, papers written in English were scanned for keywords in the titles, abstracts and list of keywords, resulting in 341 papers for further review. In the second stage, two investigators independently reviewed the full-text published papers for their relevance to the objective of this systematic review; 50 papers were included as they discussed the main challenges in the adoption of assistive robots among seniors. Subsequently, the bibliography of each selected paper was used to identify a further 20 papers that were relevant but had not been retrieved in the initial database search. A total of 70 papers were therefore selected for review (as shown in Figure 1). 


\subsection{Data Extraction and Analysis}

Two reviewers worked independently to extract the following information from the 70 papers included in the systematic review: 1) aims and objectives of the study; 2) types of assistive robots included in the study; 3) chosen study methodologies; and 4) any challenges associated with the adoption of assistive robots among seniors. Identified challenges/issues were clustered and labeled on the basis of their similarities. This assessment and labeling process was repeated iteratively until consensus was reached on cluster labels. At the end of this phase, identified challenges were then grouped into two categories related to challenges associated with adoption of 1) rehabilitation robots and 2) socially assistive robots.

\section{Results}

\subsection{Demographics of Selected Papers}

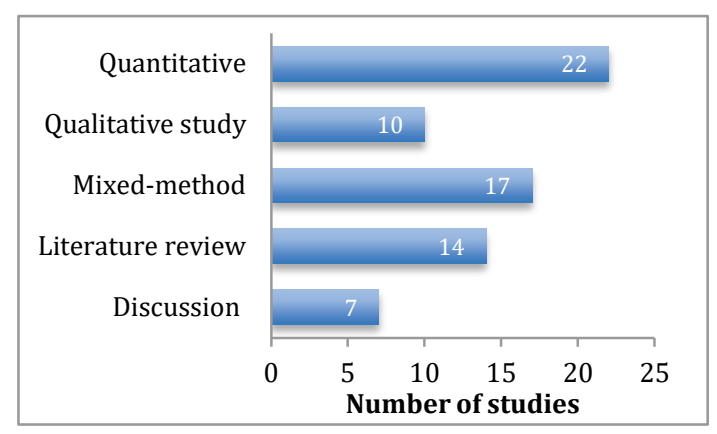

Fig. 2. Research methodologies of selected studies.

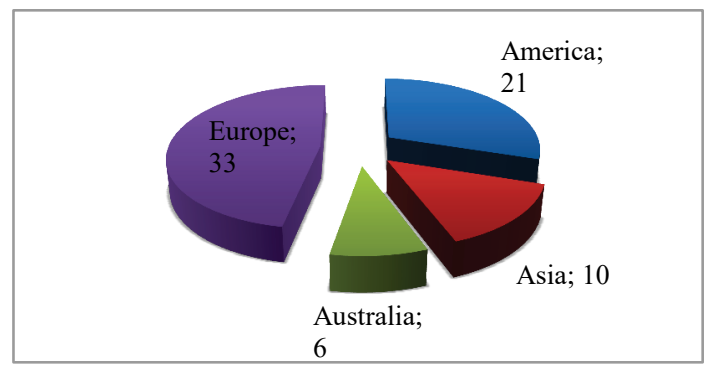

Fig. 3. Continent of origin of selected studies, showing number of studies in each area (n).

Of the selected papers $(n=70)$, the majority $(49 / 70$; $70 \%$ ) were empirical studies, followed by literature reviews and discussion papers (as shown in Figure 2). In the empirical papers, participants were asked to interact with a robot for a period of time (several minutes to months). A 'robot-acceptance measurement' was assessed via various strategies across the papers, including qualitative, quantitative and mixed-method approaches. Most of the selected papers adopted mixed- methods to address a lack of qualitative research. The papers related to studies conducted mainly in Europe $(33 / 70 ; 47 \%)$ or America $(21 / 70 ; 30 \%)$, when compared with other continents (as shown in Figure 3).

\subsection{The Challenges for Rehabilitation Robots}

As shown in Figure 4, when reviewing the challenges that affected acceptance of rehabilitation robots among seniors in these papers, multiple variables emerged, including: 1) behavioral issues; 2) technological issues; 3) costs; and 4) safety concerns.

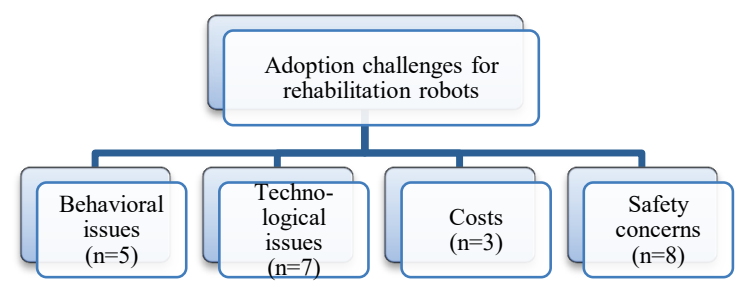

Fig. 4. The challenges in seniors adopting rehabilitation robots, showing number of studies that identified each issue (n).

\section{Behavioral Issues}

Twenty-two seniors were recruited to investigate the feasibility of hybrid assistive limb (HAL) rehabilitation. ${ }^{9}$ The HAL suit was created to support seniors with muscle weakness or impaired motor function and retrain lower extremity function following stroke. The study showed that $28 \%$ of the participants (six seniors) refused to continue and withdrew from the HAL rehabilitation program due to depression. In a different study that investigated the use of a robotic wheelchair to assist people with mobility problems, some participants felt depressed and asked for assistance in completing the study tasks. ${ }^{10}$

In contrast, a positive experience with a rehabilitation robot could effectively encourage less motivated individuals to complete their rehabilitation program. ${ }^{11-12}$ For instance, in the study conducted by Ozaki et al., ${ }^{12}$ eight older volunteers with gait disturbance were recruited to evaluate the efficacy of postural therapy with a personal transport assistance robot (PTAR); volunteers had therapeutic exercises while playing video games. Researchers believed that the combination of rehabilitation robots and video games or virtual environment could bring a sense of excitement. At the end of the study, most of the participants preferred PTAR training than traditional methods and reported a high level of enjoyment. 


\section{Technological Issues}

In the case of design issues, physical disabilities may affect individuals' capabilities with robots, for instance, in terms of auditory sensitivity, color vision, finger or hand movement control, posture and balance changes, and speed response. ${ }^{5}$ Findings have shown that seniors preferred to have rehabilitation robots with simple features to support their physical disabilities. ${ }^{5,10,13-14}$

In the case of mobility problems due to aging, seniors may benefit from power or robotic wheelchairs. ${ }^{10}$ In experiments with a robotic wheelchair called CARMEN, older volunteers stated that they could easily control the robotic wheelchair and preferred it over a conventional power wheelchair $(\mathrm{PW}) .{ }^{10}$ In another study, an intelligent PW was equipped with an obstacle avoidance feature to enable seniors to navigate and control robotic wheelchairs in crowds or small spaces, including narrow store aisles and public bathrooms. ${ }^{13}$ However, a hidden danger that clinicians are becoming concerned about is the loss of residual capabilities due to excessive assistance via robotic wheelchairs. To counter this, an adaptive collaborative control can be fitted, so disabled seniors can directly customize robotics wheelchair systems based on current health status and skill in controlling the wheelchair. ${ }^{10}$

Another crucial challenge when designing rehabilitation robots is the controller design. ${ }^{5}$ The ideal controller of rehabilitation robots should allow easy operation and support natural movements of the user, with minimal ethical consequences. ${ }^{5}$ In the case of stroke rehabilitation, the manipulator of the robot was attached to the forearms of senior participants and complex movements decomposed into sub-movements for robot control. ${ }^{14}$ As a consequence, seniors were able to perform full-range movements. ${ }^{14}$

The latest trend in rehabilitation robots is the capability of a robot to learn new techniques and to detect and recognize objects. ${ }^{5}$ Some of the latest developments are haptic interfaces, voice and facial recognition, mood and emotional state recognition. ${ }^{5}$

Future studies may be directed to the mapping of electromyographic (EMG) signals to muscle activations $^{15}$ and the development of an autonomous robot that could facilitate the special needs and preferences of seniors with cognitive decline. ${ }^{16}$

\section{Costs}

The cost of rehabilitation robots can significantly affect the adoption of technology among seniors. Some rehabilitation robots can be operated without assistance, whereas others require patients to perform prescribed treatments under direct supervision, which means additional costs for their services. ${ }^{5}$ Seniors must consider the hidden costs of having rehabilitation robots within their residential environment (for example, costs associated with installation, operation, maintenance, troubleshooting and one-on-one treatment sessions).

Lo et al. ${ }^{17}$ found the average total costs of 36 weeks of robot-assisted versus intensive therapy were $\$ 17,831$ and $\$ 19,746$, respectively. In contrast, a six-week study by Schoone et al. ${ }^{14}$ showed that a rehabilitation robot called ACRE, for enhancing upper limb therapy services, was more expensive than conventional care, at $€ 644.41$ vs. $€ 423.74$. A possible solution to this issue is to create two versions of rehabilitation robots: 1) a comprehensive and adaptable version for large rehabilitation centers and 2) a low-cost, simplified version for residential use.

\section{Safety Concerns}

One advantage of using rehabilitation robots is that a robot can provide consistent and repeatable training with minimal effort. Rehabilitation robots are created to augment, not substitute, the work of qualified therapists. Studies have highlighted the effect of robot-aided therapy on maximizing recovery from injuries ${ }^{11,18-20}$ and reducing the workload of professional therapists. ${ }^{21}$

However, regardless of the benefits, the risk of proximity accidents exists due to the fact that individuals with upper and lower extremity impairments need to have direct contact with therapy robots. When designing rehabilitation robots, the safety of users should be of utmost concern to the researchers. ${ }^{22}$ Rushton et al. ${ }^{13}$ highlighted that a PW might be removed and replaced with a manual wheelchair by caregivers due to safety concerns. Most of the participants in their study encountered accidents while driving the PW due to environmental barriers (including curbs, inaccessible buildings, uneven footpaths and uneven ground). Meadmore et al. ${ }^{23}$ reported one participant experienced shoulder muscle ache and hand and wrist pains. Researchers need to ensure that a therapy robot is designed correctly and does not permit limb movements that are outside an individual's range of motion to avoid injuries during therapy sessions. ${ }^{22}$

\subsection{The Challenges for Socially Assistive Robots}

Acceptance of socially assistive robots among seniors was affected by multiple variables, including individual characteristics, behavioral issues, technological issues (design issues, system integration, multifunctional robots and usability), costs, and privacy concerns (as shown in Figure 5). 


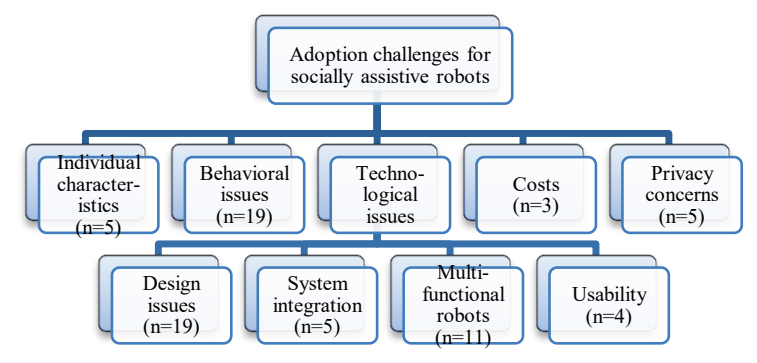

Fig. 5. The challenges in adoption of socially assistive robots, showing number of studies in each (n).

\section{Individual Characteristics}

Various studies found that age $\mathrm{ag}^{24-25}$ and gender differences in personality ${ }^{26-27}$ might be influence seniors' willingness to accept social robots in residential environments. A recent study showed that older men are eager to learn how to use either companion-type or service-type robots and had higher computer selfefficacy when compared with older women. ${ }^{27}$

A number of research papers highlighted the relationship between the educational and technological background of users and robot acceptance..$^{25,28-29}$ Sorri et al. ${ }^{29}$ reported that 'tech-savvy' seniors tend to adopt this socially assitive robot due to their advanced knowledge and experience with technology. Moreover people with higher educational backgrounds were less perceived to the existence of the robot as a social entity. ${ }^{25}$ In contrast, Louie et al. ${ }^{30}$ found no significant correlation between knowledge of computerized equipment and perceived ease of use.

\section{Behavioral Issues}

Various studies investigated positive and negative attitudes towards the use of socially assistive robots among seniors. ${ }^{31-35}$ Nomura and $\mathrm{Sasa}^{36}$ found that seniors were more open-minded when compared with university students and treated assistive robots as social entities. However, some seniors rejected the use of social robots due to uncomfortable feelings of anxiety, boredom, confusion, frustration or impatience when interacting with a social robot. ${ }^{27,33,37-39} \mathrm{Wu}$ et al. ${ }^{33}$ reported that seniors felt embarrassed and uncomfortable in using robot for assistance because they feared being labeled as handicapped.

Frail seniors were concerned about being dependent $^{40}$ or over-reliant. ${ }^{41}$ They also worried that the availability of social robots posed risks of increased loneliness, ${ }^{34}$ social isolation, ${ }^{42-45}$ inactivity or laziness. ${ }^{44}$

Additionally, Huschilt and Clune ${ }^{41}$ reported that people with dementia may become agitated and/or angry if robots malfunctioned and required repair or replacement. Some senior participants reported negative view of robots as frightening or dangerous in another study. $^{34}$

Countering these negative situations, Beer et al. ${ }^{46}$ recommended adding features to establish dynamic interactions between assistive robots and users. Seniors could maintain active lifestyles and cope with their limitations via assistance from the robots. Other studies also have emphasized the importance of having a robot that can be adjusted based on individuals needs and performances, since age-related health decline may affect the physical function of seniors. ${ }^{27}$

User attitudes may also have be affected by feedback from family members or caregivers. Broadbent et al. $^{2}$ reported that healthcare workers expressed concerns around losing their jobs because of assistive robots, and family members of seniors felt uneasy about the possibility of workers being replaced by robots. The same study found limited knowledge of robotic technology could cause job insecurity among healthcare professionals. In addition, both caregivers and relatives may have doubts about the quality of healthcare services provided by a robot. In that situation, family caregivers felt robots must not replace humans when performing care activities and should only be considered as a 'helper'. ${ }^{35}$

\section{Technological Issues}

\section{- Design issues}

Several studies found that seniors' viewpoints were more polarized when discussing features of robots. Seniors have different impressions of preferred robot size; a small robot can create a tripping hazard. ${ }^{2}$ while a larger robot may not fit in small areas. ${ }^{44,47-50}$ A midsized robot was preferred, with the height between 1.25 $\mathrm{m}$ and $1.50 \mathrm{~m}^{2,51}$ In addition, robots must be sufficiently stable and reliable to perform different kinds of activities ${ }^{51}$ and to provide support for seniors who need assistance in maintaining balance, in the case of slips and trips. ${ }^{2,44}$ Broadbent et al. ${ }^{2}$ stated that the preferred colors were silver, gold or white.

The shape of robots is another topic of discussion in. ${ }^{2,39.47}$ Hutson et al. ${ }^{47}$ found some seniors were dissatisfied with pet-like companionship robots and expressed high expectations that a robot would mimic real behavior of an animal. Seniors also preferred to have a machine-like robot rather than a humanoid robot, ${ }^{2}$ perhaps because seniors saw robots as non-living mechanical objects that should not replace a human presence. In contrast, seniors in a different study preferred to interact with a robot that was more human in shape. ${ }^{28}$

Socially assistive robots for seniors must include a verbal communication feature for a sense of 
togetherness. ${ }^{52}$ Some seniors have difficulty hearing a synthesized voice ${ }^{37}$ and preferred a male rather than female voice. ${ }^{2,27}$ The voice of a robot should be clear, 2,50 soft $^{49}$ and have a neutral accent. ${ }^{53-53}$ In addition, improper audio transmission from a robot might cause interference with hearing aids. ${ }^{55}$

Other things to consider when designing socially assistive robots are: clearly visible screens, which can be activated when needed; ${ }^{2}$ large buttons; ${ }^{2,50}$ larger icons without text displayed on the screen rather than smaller icons with the app names; ${ }^{56}$ and capability to recognize speech commands under noisy conditions. ${ }^{2,57}$

\section{- System integration}

To be adopted, technologies must be capable of fitting seamlessly into daily living activities, including for seniors. $^{25,40,58}$ Seniors showed a preference for robots that can be adjusted to suit individual skills and needs, ${ }^{36,45}$ appropriate when age-related health decline may affect the physical and psychological status of older adults. ${ }^{37}$ To address this, one study proposed a semi-automatic adjustment mechanism for assistive robots so that users can have full control. ${ }^{59}$

\section{- Multi-functional robots}

Most socially assistive robots can act as either a servicetype robot or a companionship robot, but not a combination of two. However, it must be considered that seniors require both care assistance and social interactions. ${ }^{60}$ Thus, features of an ideal robot would include: an alert system, ${ }^{35,61}$ fall detection system, ${ }^{4,51}$ medication reminder ${ }^{62}$ and multimedia messenger. ${ }^{63}$

A project called "Domeo" aimed to ensure seniors could remain at home for longer by using a service-type robot (called "Kompai") for care assistance. ${ }^{35}$ The functionalities of the robot connected the user with their family through verbal and visual interactions, sending a video stream in the case of dangerous situations, providing stimulation when the user performed physical exercises, and observing the user's behavior. ${ }^{35}$

Cesta et al. $^{64}$ reported a positive reaction from seniors regarding the use of a proactive robot in emergency situations. In contrast, Zsiga et al. ${ }^{35}$ found that seniors showed some concerns related to slow response of robots during a dangerous situation.

In the case of medication reminder, Prakash et al. ${ }^{62}$ outlined that even though seniors preferred to be reminded about medication by a robot, they still demanded assistance with taking medication to preempt, and prevent, potential mistakes by robots.

Several studies have highlighted the importance of robot butlers or robot servants in the daily activities of seniors. ${ }^{45,51,61,63}$ However, there are some issues with household robots that should be anticipated, for instance, failure of the robot's operation could damage the residence. ${ }^{46}$ Regardless of potential benefits offered by socially assistive robots, seniors argued that a robot is not needed to manage daily activities, ${ }^{34}$ since those functionalities are already provided by other, existing commercial products, such as mobile phones and global positioning systems (GPS).

\section{- Usability}

Few studies have emphasized the importance of developing an assistive robot that was easy to use for seniors, with or without technical knowledge. ${ }^{25,27,41,48}$ Frennert et al. ${ }^{44}$ suggested that an assistive robot may not be easy to use by seniors, especially if a user has a history of severe cognitive impairment, which could lead to inability to learn new things.

Seelye et al. ${ }^{61}$ proposed using a telepresence robot to enable active communication between seniors and their relatives. Findings from the study were that some participants felt slightly confused when operating handheld remotes and one participant with mild cognitive impairment found that controlling the robot was sometimes quite overwhelming due to its complexity.

\section{Costs}

The current cost to purchase, install and manage a robot is still quite expensive, ${ }^{32,66}$ beyond the capacity of many seniors, who tend to carefully consider financial decisions. $^{65}$ It must be understood that immediate benefits of robotic technologies are few and future costsaving cannot be forecast in the short term. ${ }^{66}$ In other words, technology adoption can be hindered by an imbalance between real costs and prospective values.

\section{Privacy Concerns}

Privacy concerns are heightened in settings for seniors, such as a surveillance robot in a residential environment $^{61}$ or retirement village. ${ }^{2}$ For example. Broadbent and colleagues ${ }^{2}$ reported that most older participants in their study rejected the use of a robot for continuous monitoring activities. In addition, Feil-Seifer et al. ${ }^{42}$ demonstrated that an accidental violation of privacy may occur when an assistive robot is incapable of differentiating between confidential data and general information shared by a user. This is due to the fact that older participants were not fully aware about information collected from their residential environment $^{67}$ and the fact that the system may or may not be accessed by users. ${ }^{31}$

Autonomy must be granted to users to solve these privacy concerns. ${ }^{61}$ Seniors should be able to decide who can directly contact them, for example, through mobile robotic telepresence, as demonstrated in the ORCATECH Living Laboratory project. ${ }^{61}$ Moreover, it 
was recommended that a surveillance robot must be accompanied by a specific formulated policy to ensure privacy rights of seniors are protected within a residential environment. ${ }^{67}$

\section{Discussion}

Research has shown that the growth of assistive robots for seniors is inevitable and can provide significant changes in the delivery of rehabilitation ${ }^{19-20}$ and wellbeing services. ${ }^{43}$ Although assistive robots are designed so that features are most useful for end users, it must be noted that failure to adopt a robot may be due to social stigmatization or a lack of understanding of seniors' needs and preferences for assistive robots. Based on our review, only $14.3 \%$ of the selected studies included a qualitative evaluation of acceptance of assistive robots among seniors. Qualitative studies can provide more information with respect to the nuances and context of particular problems in this area. Moreover, only 14.3\% of the selected studies were conducted in Asian countries. We believe that this geographical distribution reflects the fact that Westerners tend to adopt the use of robots around residential and/or aged care facilities. Further studies in developing countries are required to investigate how culture affects adoption of assistive robots among seniors.

\subsection{Rehabilitation Robots}

In the case of rehabilitation robots, the key driver among seniors is their perceived value of technology, since their decisions are often based solely on desired impacts. A number of studies reviewed indicated that the use of robot-assisted therapy for upper-limb rehabilitation lead to improvements of the hand, wrist and elbow motor functions. ${ }^{19-20}$ Conversely, a study by Pang et al. $^{68}$ revealed that seniors with severe impairment of upper extremity function showed no improvement even after attending 57 robot-assisted therapy sessions over a 19 -week period.

Moreover, it is highly preferable to build a user interface that is easy to operate and that provides more natural and friendly man-machine interactions for better acceptance. ${ }^{69}$ Nowadays, developing user interfaces that address the varying needs of different users is completed via trial and error. There is therefore a need to implement artificial intelligence in rehabilitation robots that automatically tailor the rehabilitation process to the needs and specific physical conditions of disabled seniors. ${ }^{5}$ In addition, more natural interactions between human and rehabilitation robots can be achieved by equipping the robots with short-term memory, longterm storage, words and language databases. ${ }^{5}$
To minimize risk of impairments and injuries, recent designs have focused on constructing low-power, lightweight rehabilitation robots to allow users to perform therapeutic movements at a preferred pace. ${ }^{5}$ Moreover, these robots have built in backup and restore functionalities in the case of a malfunction. ${ }^{5}$ However, Bemelmans et al. $^{70}$ reported that these additional features still do not guarantee a $100 \%$ level of safety. Additionally, rehabilitation robots should be capable of self-learning and self-adaptation so that they can deal with unexpected situations and can be fitted specifically to each individual's special needs. ${ }^{5}$

Clearly many questions have been left unanswered in the literature. For instance, the efficacy of robotassisted rehabilitation therapy when compared with conventional therapy. More studies are needed to determine whether conventional therapy is comparable or superior to robot-assisted therapy and whether a combination of the two methods would achieve optimal rehabilitation outcomes.

To elicit the best outcomes of rehabilitation, some studies have suggested combining rehabilitation robots with motivational games, to increase a patient's willingness to complete rehabilitation processes. ${ }^{11-12}$ The challenges and difficulty level in motivational games must gradually increase to maintain the interest and motivation of patients throughout rehabilitation sessions. ${ }^{71}$ Furthermore, healthcare staff can incorporate rewards or acknowledgements every time a patient completes games. ${ }^{71}$ This form of positive reinforcement allows patients to feel satisfied and accomplished. ${ }^{71}$ Also, they are more likely to get more intensive therapy sessions. $^{71}$

One of most interesting findings of this review was that in socially assistive robots, privacy issues and individual characteristics are of interest, while this is not the case for rehabilitation robots. We believe this to be expected and this idea has been noted previously in adoption of assistive technologies for aged care. For example, adoption could be related to context whereby a person with chronic disease may deliberately adopt technology to help manage his/her conditions. If a person is suffering a chronic disease (for example dementia), they will be much more open to adopting and using rehabilitation robots for cognitive therapy. ${ }^{16}$ In a study conducted by Schoone et al., ${ }^{14}$ therapeutic treatment of upper limbs was performed using the ACRE robotic device while a therapist sat next to participants to ensure the therapy treatment was safe and effective. This means seniors without prior knowledge of rehabilitation robots were still able to perform rehabilitative training through the use of a robot.

Finally, this systematic review revealed that most of the selected publications are focused on the socially assistive robots (50 out of 70 studies). We suggest 
researchers could conduct more studies in system integration and usability of rehabilitation robots as perceived by seniors, and the relationship between acceptance of rehabilitation robots and demographic attributes of seniors (age, educational and technological background, and gender).

\subsection{Socially Assistive Robots}

In the case of socially assistive robots, Sharkey et al. ${ }^{43}$ expressed concerns that the use of assistive robots could lead to ethical issues. Specifically, the reduction in social contact between seniors and their family members, and the potential isolation and neglect of seniors by communities may be more prevalent than before. ${ }^{43}$ This situation could happen if family members start to believe that robots can provide both physical and emotional supports for seniors and therefore neglect their parents. Family members should understand that these robots provide many benefits, but cannot substitute interpersonal relationships and social connections.

In term of cost and workforce issues, seniors and their family members may want to consider the following few points before adopting socially assistive robots: the cost effectiveness of using those robots; the workforce reduction in the healthcare industry; and the workforce demand in a technical field. ${ }^{72}$ First of all, they need to compare the costs that would have been involved if they chose to use socially assistive robots rather than to employ healthcare staff. ${ }^{72}$ The cost of using robots would include: cost of home modification; installation cost; recurring costs, such as electricity and Internet bills, ongoing maintenance/ troubleshooting, subscription fees; and hidden costs, such as insurance for faulty robots leading to damage, bodily harm, or impairment of quality of life. Secondly, they also need to consider an alternative solution beside socially assistive robots to solve the potential healthcare workforce shortage. ${ }^{72}$ As an illustration, they may need to take account of the benefits of recruiting, for example, unemployed youth for senior companionship. The younger generation may relish such jobs and the required skills can be obtained, developed and improved through training courses in aged care services.

Socially assistive robots can also be used to ease the burden on nursing staff, for instance: to safely move a senior stroke survivor from a bed to a wheelchair ${ }^{73}$ to safely guide a senior person with vision impairment to move around their house or aged care facility; ${ }^{74}$ or to improve brain functionality for seniors with dementia by accompanying them to play memory games. ${ }^{27}$ There are certain situations and case scenarios where socially assistive robots may be more desirable than healthcare professionals, for instance, some careers may lose their patience or temper more frequently when dealing with ornery people, while a robot will not. ${ }^{72}$ By collaborating with professional staff, socially assistive robots can effectively maintain the health, safety and wellbeing of seniors. These professional staff could still be responsible for remotely controlling and monitoring the functionality of semi-autonomous robots via an Internet connection. $^{72}$

The sustainability of socially assistive robots is the third aspect that needs to be considered when placing robots in non-technical environments. ${ }^{72}$ Some houses are not equipped for high technology support and may be located in rural areas. ${ }^{72}$ In that situation, technicians would need to travel quite long distances for installation, ongoing maintenance and troubleshooting. ${ }^{72}$ The applicable robotic system must be designed and constructed so that the technicians can remotely configure and troubleshoot the system over the Internet after the completion of installation. ${ }^{72}$

In terms of privacy considerations, it is crucial that full autonomy be granted to the user to avoid any intrusions on the privacy data of seniors. ${ }^{42}$ All sensitive data related to senior volunteers in clinical studies (for instance, identities and health records) must be stored securely and could only be accessed by authorized parties. $^{42}$

The above-discussed challenges are coupled with the fact that many robot developers have deep-rooted, stereotypical views of seniors as frail or senile, which makes them unable to design assistive robots that fulfill the real needs of seniors. ${ }^{75}$ This view contrasts with the reality that a heterogeneous group of seniors may include many 'technogenarians', when considering their age and sex, level of education, an previous experience with technology. ${ }^{25,27,29}$ Robotic researchers and developers need to be more open-minded about the diversity of older generations ${ }^{75}$ and involve them in the development process for better acceptance during implementation. ${ }^{32,76}$

One of the most important factors that influences seniors' decisions whether to accept the existence of a robot in the residential environment is the robot's potential benefits. ${ }^{24}$ Researchers and developers need to highlight the advantages of using a robot and provide a series of enjoyable training sessions for senior users. ${ }^{77}$ For instance, the expected benefits of using socially assistive robots are to support independence and give meaning, ${ }^{35}$ provide comfort and safety for seniors and their family members, ${ }^{35}$ minimize loneliness, ${ }^{61}$ reduce the incidence of skipped medication, ${ }^{62}$ and improve awareness after falls or injuries. ${ }^{4,51}$ So then, in the future, it will greatly enhance the user acceptance and adoption of robots. 


\section{Study Limitations}

This review, like any literature review, is highly dependent on the keywords that were chosen and the databases selected. To minimize this, we conducted a background and exploratory study prior to our review to ensure selection bias was avoided as much as possible. In addition, because our reviews embraced complexity and sought to map out the operation of multiple challenges, the search was quite broad. However, we could still only cover a limited number of papers, published between 2005 and 2016, inclusive. The selected papers also only considered the seniors' perspectives toward the concept of using assistive robots to improve their quality of life. Although this decision was made in order to focus on the study of recent developments in the field, it is still arguable that authors may have missed valuable robot adoption approaches that are applicable to today's older population.

Future research could also include papers that examined the perspectives of seniors' family members and healthcare providers towards the use of assistive robots for seniors. Family members and healthcare providers are actively involved in providing care to seniors and their feedback can be useful to developing and adopting assistive robots in residential and aged care settings. Moreover, before implementing assistive robots in aged care systems, it is important to completely understand the aged care workflow and how assistive robots can be used to improve the effectiveness of delivering aged care services worldwide.

\section{Conclusion}

This systematic review provides an in-depth understanding of challenges related to the adoption of assistive robots among seniors. Our review highlights that the availability of technologies that indirectly portray seniors as frail and requiring continuous care can be a major factor in why seniors are not ready to adopt robotic technology. Assistive robots that target seniors must preserve the dignity of seniors as well as prolong their independence. ${ }^{78}$ We believe that the challenges of adopting assistive robotic technologies among seniors were more significantly related to sociotechnical $^{79}$ and economical ${ }^{32}$ aspects rather than individual characteristics like age and gender.

The knowledge gained from this review will be valuable for system designers and developers, specifically regarding assistive robots to accommodate the needs of seniors with and without physical and psychological impairments. Furthermore, this systematic review can disseminate information to therapists and healthcare staff on salient factors that affect seniors adopting assistive robots. It is hoped that healthcare professionals will be able to select the right robot based on the current health status and needs of seniors and provide more feedback to developers and researchers to improve design of assistive robots.

Currently, our research group is investigating the barriers and driving factors when implementing a telepresence system to care for seniors, which consists of mobile robotic telepresence, a fall detector and a medication adherence device. It is expected that the outcomes of the project will lead to better awareness and acceptance of assistive technologies within this aging population.

\section{Acknowledgements}

The research reported in this paper is a part of the Australian contribution to the EU "Victoryahome" project.

\section{References}

1. W. Swan, Australia to 2050: future challenges, (Commonwealth of Australia, 2010).

2. E. Broadbent, R. Tamagawa, A. Patience, B. Knock, N. Kerse, K. Day, and B.A. MacDonald, 'Attitudes towards health-care robots in a retirement village', Australasian Journal on Ageing, vol. 31, no. 2, 2012, pp. 115-120.

3. D. Feil-Seifer, and M.J. Mataric, 'Defining socially assistive robotics', In: Proceedings of the 9th International Conference on Rehabilitation Robotics, 2005, pp. 465-468.

4. J. Broekens, M. Heerink, and H. Rosendal, 'Assistive social robots in elderly care: a review', Gerontechnology, vol. 8, no. 2, 2009, pp. 94-103.

5. F. Yakub, A.Z.M. Khudzari, and Y. Mori, 'Recent trends for practical rehabilitation robotics, current challenges and the future', International Journal of Rehabilitation Research, vol. 37, no. 1, 2014, pp. 9-21.

6. Y-H. Wu, V. Faucounau, M. Boulay, M. Maestrutti, and A.-S. Rigaud, 'Robotic agents for supporting communitydwelling elderly people with memory complaints, perceived needs and preferences', Health Informatics Journal, vol. 17, no. 1, 2011, pp. 33-40.

7. K. Gurley, and A.F. Norcio, 'A systematic review of technologies designed to improve and assist cognitive decline for both the current and future aging populations', In: Proceedings of the Internationalization, Design and Global Development, 2009, pp. 156-163.

8. D. Moher, A. Liberati, J. Tetzlaff, and D.G. Altman, 'Preferred reporting items for systematic reviews and meta-analyses: the PRISMA statement', Annals of Internal Medicine, vol. 151, no. 4, 2009, pp. 264-269.

9. T. Ueba, O. Hamada, T. Ogata, T. Inoue, E. Shiota, and Y. Sankai, 'Feasibility and safety of acute phase rehabilitation after stroke using the hybrid assistive limb robot suit', Neurologia Medico-Chirurgica, vol. 53, no. 5 2012, pp. 287-290. 
10. C. Urdiales, B. Fernandez-Espejo, R. Annicchiaricco, F. Sandoval, and C. Caltagirone, 'Biometrically modulated collaborative control for an assistive wheelchair', IEEE Transactions on Neural Systems and Rehabilitation Engineering, vol. 18, no. 4, 2010, pp. 398-408.

11. R.A. Cooper, B.E. Dicianno, B. Brewer, E. LoPresti, D. Ding, R. Simpson, G. Grindle, and H. Wang, 'A perspective on intelligent devices and environments in medical rehabilitation', Medical Engineering and Physics, vol. 30, no. 10, 2008, pp. 1387-1398.

12. K. Ozaki, H. Kagaya, S. Hirano, I. Kondo, S. Tanabe, N. Itoh, E. Saitoh, T. Fuwa, and R. Murakami, 'Preliminary trial of postural strategy training using a personal transport assistance robot for patients with central nervous system disorder', Archives of Physical Medicine and Rehabilitation, vol. 94, no. 1, 2013, pp. 59-66.

13. P. W. Rushton, D. Kairy, P. Archambault, E. Pituch, C. Torkia, A. El Fathi, ... and L. Demers, 'The potential impact of intelligent power wheelchair use on social participation: perspectives of users, caregivers and clinicians', Disability and Rehabilitation: Assistive Technology, vol. 10, no. 3, 2014, pp. 191-197.

14. M. Schoone, E. Dusseldorp, M.E. van den Akker-van Marle, A.J. Doornebosch, R. Bal, A. Meems, M. Oderwald, and R. van Balen, 'Stroke rehabilitation in frail elderly with the robotic training device ACRE: a randomized controlled trial and cost-effectiveness study', Journal of Robotics, vol. 2011, no. 543060, 2011, pp. 110.

15. S.K. Ho, Chapter 6. Hand rehabilitation robot using electromyography (EMG), In: Biomechatronics in Medicine and Health Care (Pan Stanford Publishing, Singapore, 2011), pp. 77-92.

16. F. Martín, C. Agüero, J.M. Cañas, G. Abella, R. Benítez, S. Rivero, M. Valenti, and P. Martínez-Martín, 'Robots in therapy for dementia patients', Journal of Physical Agents, vol. 7, no. 1, 2013, pp. 48-55.

17. A.C. Lo, P.D. Guarino, L.G. Richards, J.K. Haselkorn, G.F. Wittenberg, D.G. Federman, ... and P. Peduzzi, 'Robot-assisted therapy for long-term upper-limb impairment after stroke', New England Journal of Medicine, vol. 362, no. 19, 2010, pp. 1772-1783.

18. F. Bovolenta, M. Goldoni, P. Clerici, M. Agosti, and M. Franceschini, 'Robot therapy for functional recovery of the upper limbs: a pilot study on patients after stroke', Journal of Rehabilitation Medicine, vol. 41, no. 12, 2009, pp. 971-975.

19. O. Lambercy, L. Dovat, H. Yun, S.K. Wee, C.W. Kuah, K.S. Chua, ... and E. Burdet, 'Effects of a robot-assisted training of grasp and pronation/supination in chronic stroke: a pilot study', Journal of Neuroengineering and Rehabilitation, vol. 8, no. 1, 2011, pp. 63.

20. X.L. Hu, K.Y. Tong, R. Li, J.J. Xue, S.K. Ho, and P. Chen, 'The effects of electromechanical wrist robot assistive system with neuromuscular electrical stimulation for stroke rehabilitation', Journal of
Electromyography and Kinesiology, vol. 22, no. 3, 2012, pp. 431-439.

21. A.M. Okamura, M.J. Mataric, and H.I. Christensen, 'Medical and health-care robotics', Robotics and Automation Magazine, vol. 17, no. 3, 2010, pp. 26-27.

22. A. Wynsberghe, Designing robots with care: Creating an ethical framework for the future design and implementation of care robots, Ph.D. thesis, Department of Philosophy, Universiteit Twente, 2012.

23. K.L. Meadmore, A.M. Hughes, C.T. Freeman, V. Benson, and J.H. Burridge, 'Participant feedback in the evaluation of novel stroke rehabilitation technologies', Journal of Rehabilitation Robotics, vol. 1, no. 2, 2013, pp. 82-92.

24. N. Ezer, A.D. Fisk, and W.A. Rogers, 'Attitudinal and intentional acceptance of domestic robots by younger and older adults', In: Universal access in human-computer interaction. Intelligent and Ubiquitous Interaction Environments (Springer Berlin Heidelberg, 2009), pp. 3948.

25. M. Heerink, 'Exploring the influence of age, gender, education and computer experience on robot acceptance by older adults', In: Proceedings of the 6th ACM/IEEE International Conference on Human-Robot Interaction, 2011a, pp. 147-148.

26. I.H. Kuo, J.M. Rabindran, E. Broadbent, Y.I. Lee, N. Kerse, R.M.Q. Stafford, and B.A. MacDonald, 'Age and gender factors in user acceptance of health-care robots', In: Proceedings of the 18th IEEE International Symposium on Robot and Human Interactive Communication, 2009, pp. 214-219.

27. W.Y.G. Louie, D. McColl, and G. Nejat, 'Acceptance and attitudes toward a human-like socially assistive robot by older adults', Assistive Technology, vol. 26, no. 3, 2014, pp. 140-150.

28. M. Scopelliti, M.V. Giuliani, and F. Fornara, 'Robots in a domestic setting: a psychological approach', Universal Access in the Information Society, vol. 4, no. 2, 2005, pp. 146-155.

29. L. Sorri, and E. Leinonen, 'Technology that persuades the elderly', In: Persuasive Technology (Springer Berlin Heidelberg, 2008), pp. 270-273.

30. W.Y.G. Louie, D. McColl, and G. Nejat, 'Playing a memory game with a socially assistive robot: a case study at a long-term care facility', In: Proceedings of the $21 \mathrm{st}$ IEEE International Symposium on Robot and Human Interactive Communication, 2012, pp. 345-350.

31. A. Kristoffersson, S. Coradeschi, A. Loutfi, and K. Severinson-Eklundh, 'An Exploratory Study of Health Professionals' attitudes about robotic telepresence technology', Journal of Technology in Human Services, vol. 29, no. 4, 2011, pp. 263-283.

32. Y.H. Wu, C. Fassert, and A.S. Rigaud, 'Designing robots for the elderly: appearance issue and beyond', Archives of Gerontology and Geriatrics, vol. 54, no.1, 2012, pp. 121126. 
33. Y.H. Wu, J. Wrobel, M. Cornuet, H. Kerhervé, S. Damnée, and A.S. Rigaud, 'Acceptance of an assistive robot in older adults: a mixed-method study of humanrobot interaction over a 1-month period in the Living Lab setting', Clinical Interventions in Aging, vol. 9, no. 1, 2014, pp. 801-811.

34. Y.H. Wu, V. Cristancho-Lacroix, C. Fassert, V. Faucounau, J. de Rotrou, and A.S. Rigaud, 'The attitudes and perceptions of older adults with mild cognitive impairment toward an assistive robot', Journal of Applied Gerontology, vol. 35, no. 1, 2016, pp. 3-17.

35. K. Zsiga, G. Edelmayer, P. Rumeau, O. Péter, A. Tóth, and G. Fazekas, 'Home care robot for socially supporting the elderly: focus group studies in three European countries to screen user attitudes and requirements', International Journal of Rehabilitation Research, vol. 36, no. 4, 2013, pp. 375-378.

36. T. Nomura, and M. Sasa, 'Investigation of differences on impressions of and behaviors toward real and virtual robots between elder people and university students', In: Proceedings of the 11th IEEE International Conference on Rehabilitation Robotics, 2009, pp. 934-939.

37. J. Fasola, and M.J. Mataric, 'Robot exercise instructor: A socially assistive robot system to monitor and encourage physical exercise for the elderly', In: Proceedings of the 19th IEEE International Symposium on Robot and Human Interactive Communication, 2010, pp. 416-421.

38. G. Nejat, M.A. Nies, and T.R. Sexton, 'An interdisciplinary team for the design and integration of assistive robots in health care applications', Home Health Care Management and Practice, vol. 22, no. 2, 2010, pp. 104-110.

39. M. D'iaz, J. Saez-Pons, M. Heerink, and C. Angulo, 'Emotional factors in robot-based assistive services for elderly at home', In: Proceedings of the 22nd IEEE International Symposium on Robot and Human Interactive Communication, 2013, pp. 711-716.

40. A. Cesta, G. Cortellessa, V. Giuliani, F. Pecora, M. Scopelliti, and L. Tiberio, 'Psychological implications of domestic assistive technology for the elderly', PsychNology Journal, vol. 5, no. 3, 2007a, pp. 229-252.

41. J. Huschilt, and L. Clune, 'The use of socially assistive robots for dementia care', Journal of Gerontological Nursing, vol. 38, no. 10, 2012, pp. 15-19.

42. D. Feil-Seifer, and M.J. Mataric, 'Socially assistive robotics', IEEE Robotics and Automation Magazine, vol. 18 , no. 1, 2011, pp. 24-31.

43. A. Sharkey, and N. Sharkey, 'Granny and the robots: ethical issues in robot care for the elderly', Ethics and Information Technology, vol. 14, no. 1, 2012, pp. 27-40.

44. S. Frennert, H. Eftring, and B. O" stlund, 'Older people's involvement in the development of a social assistive robot', Social Robotics, vol. 8239, no. 1, 2013, pp. 8-18.

45. T. Sorell, and H. Draper, 'Robot carers, ethics, and older people', Ethics and Information Technology, vol. 16, no. 3, 2014, pp. 183-195.
46. J.M. Beer, C.A. Smarr, T.L. Chen, A. Prakash, T.L. Mitzner, C.C. Kemp, and W.A. Rogers, 'The domesticated robot: design guidelines for assisting older adults to age in place', In: Proceedings of the 7th ACM/IEEE International Conference on Human-Robot Interaction, 2012, pp. 335-342.

47. S. Hutson, S.L. Lim, P.J. Bentley, N. Bianchi-Berthouze, and A. Bowling, 'Investigating the suitability of social robots for the wellbeing of the elderly', Affective Computing and Intelligent Interaction, vol. 6974, no. 1, 2011, pp. 578-587.

48. C.H. King, T.L. Chen, Z. Fan, J.D. Glass, and C.C. Kemp, 'Dusty: an assistive mobile manipulator that retrieves dropped objects for people with motor impairments', Disability and Rehabilitation: Assistive Technology, vol. 7, no. 2, 2012, pp. 168-179.

49. M. Begum, R. Wang, R. Huq, and A. Mihailidis, 'Performance of daily activities by older adults with dementia: the role of an assistive robot', In: Proceedings of the 2013 IEEE International Conference on Rehabilitation Robotics, 2013, pp. 1-8.

50. F. Cavallo, M. Aquilano, M. Bonaccorsi, R. Limosani, A. Manzi, M. Carrozza, and P. Dario, 'On the design, development and experimentation of the Astro assistive robot integrated in smart environments', In Proceedings of the 2013 IEEE International Conference on Robotics and Automation, 2013, pp. 4310-4315.

51. E. Broadbent, R. Tamagawa, N. Kerse, B. Knock, A. Patience, and B. MacDonald, 'Retirement home staff and residents' preferences for healthcare robots', In: Proceedings of the 18th IEEE International Symposium on Robot and Human Interactive Communication, 2009, pp. 645-650.

52. Y. Iwamura, M. Shiomi, T. Kanda, H. Ishiguro, and N. Hagita, 'Do elderly people prefer a conversational humanoid as a shopping assistant partner in supermarkets?', In: Proceedings of the 6th ACM/IEEE International Conference on Human-Robot Interaction, 2011, pp. 449-457.

53. R. Khosla, M.T. Chu, and K. Nguyen, 'Affective robot enabled capacity and quality improvement of nursing home aged care services in Australia', In: Proceedings of the 37th Annual Computer Software and Applications Conference Workshops, 2013a, pp. 409-414.

54. R. Khosla, M.T. Chu, and K. Nguyen, 'Enhancing emotional well being of elderly using assistive social robots in Australia', In: Proceedings of the 2013 International Conference on Biometrics and Kansei Engineering, 2013b, pp. 41-46.

55. D. McColl, W.Y.G. Louie, and G. Nejat, 'Brian 2.1: A socially assistive robot for the elderly and cognitively impaired', IEEE Robotics and Automation Magazine, vol. 20, no. 1, 2013, pp. 74-83.

56. C. Granata, M. Chetouani, A. Tapus, P. Bidaud, and V. Dupourqu, 'Voice and graphical-based interfaces for interaction with a robot dedicated to elderly and people with cognitive disorders', In: Proceedings of the 19th 
IEEE International Symposium on Robot and Human Interactive Communication, 2010, pp. 785-790.

57. C. Schroeter, S. Mueller, M. Volkhardt, E. Einhorn, C. Huijnen, H. van den Heuvel, ... and H.M. Gross, 'Realization and user evaluation of a companion robot for people with mild cognitive impairments', In: Proceedings of the 2013 IEEE International Conference on Robotics and Automation, 2013, pp. 1153-1159.

58. R. Bogue, 'Robots to aid the disabled and the elderly', Industrial Robot: An International Journal, vol. 40, no. 6, 2013, pp. 519-524.

59. M. Heerink, 'How elderly users of a socially interactive robot experience adaptiveness, adaptability and user control', In: Proceedings of the 12th International Symposium on Computational Intelligence and Informatics, 2011b, pp. 79-84.

60. A.J. Pearce, B. Adair, K. Miller, E. Ozanne, C. Said, N. Santamaria, and M.E. Morris, 'Robotics to enable older adults to remain living at home', Journal of Aging Research, vol. 2012, no. 538169, 2012, pp. 1-10.

61. A.M. Seelye, K.V. Wild, N. Larimer, S. Maxwell, P. Kearns, and J.A. Kaye, 'Reactions to a remote-controlled video-communication robot in seniors' homes: a pilot study of feasibility and acceptance', Telemedicine and eHealth, vol. 18, no. 10, 2012, pp. 755-759.

62. A. Prakash, J.M. Beer, T. Deyle, C.A. Smarr, T.L. Chen, T.L. Mitzner, ... and W.A. Rogers, 'Older adults' medication management in the home: how can robots help?', In: Proceedings of the 8th ACM/IEEE International Conference on Human-Robot Interaction, 2013, pp. 283-290.

63. D.O. Johnson, R.H. Cuijpers, J.F. Juola, E. Torta, M. Simonov, A. Frisiello, ... and C. Beck, 'Socially Assistive Robots: A comprehensive approach to extending independent living', International Journal of Social Robotics, vol. 6, no. 2, 2014, pp. 195-211.

64. A. Cesta, G. Cortellessa, V. Giuliani, F. Pecora, R. Rasconi, M. Scopelliti, and L. Tiberio, 'Proactive assistive technology: An empirical study', In: HumanComputer Interaction-INTERACT 2007 (Springer Berlin Heidelberg, 2007b), pp. 255-268.

65. J.M. Beer, and L. Takayama, 'Mobile remote presence systems for older adults: acceptance, benefits, and concerns?', In: Proceedings of the 6th International Conference on Human-Robot Interaction, 2011, pp. 1926.

66. T. Blackman, 'Care robots for the supermarket shelf: a product gap in assistive technologies', Ageing and Society, vol. 33, no. 5, 2013, pp. 763-781.

67. K. Caine, S. Šabanović, and M. Carter, 'The effect of monitoring by cameras and robots on the privacy enhancing behaviors of older adults?', In: Proceedings of the 7th ACM/IEEE International Conference on HumanRobot Interaction, 2012, pp. 343-350.
68. M.Y. Pang, J.E. Harris, and J.J. Eng, 'A communitybased upper-extremity group exercise program improves motor function and performance of functional activities in chronic stroke: a randomized controlled trial', Archives of Physical Medicine and Rehabilitation, vol. 87, no. 1, 2006, pp. 1-9.

69. Z. Qian, and Z. Bi, 'Recent development of rehabilitation robots', Advances in Mechanical Engineering, vol. 7, no. 2, 2015, pp. 563062.

70. R. Bemelmans, G.J. Gelderblom, P. Jonker, and L. De Witte, 'Socially assistive robots in elderly care: A systematic review into effects and effectiveness', Journal of the American Medical Directors Association, vol. 13, no. 2, 2012, pp. 114-120.

71. A. Weightman, M.A.C. Alexoulis-Chrysovergis, and M.S. Oltean, 'What should we consider when designing rehabilitation robots for the upper limb of the neurologically impaired?', In: Proceedings of the Australasian Conference on Robotics and Automation, 2014, pp. 1-10.

72. T.S. Dahl, and M.N.K. Boulos, 'Robots in Health and Social Care: A Complementary Technology to Home Care and Telehealthcare?', Robotics, vol. 3, no. 1, 2013, pp. 1-21.

73. T. Mukai, S. Hirano, H. Nakashima, Y. Kato, Y. Sakaida, S. Guo, and S. Hosoe, 'Development of a nursing-care assistant robot Riba that can lift a human in its arms', In: Proceedings of the 2010 IEEE/RSJ International Conference on Intelligent Robots and Systems, 2010, pp. 5996-6001.

74. G.J. Lacey, and D. Rodriguez-Losada, 'The evolution of Guido', IEEE Robotics \& Automation Magazine, vol. 15, no. 4, 2008, pp. 75-83.

75. S. Frennert, and B. Östlund, 'Review: seven matters of concern of social robots and older people', International Journal of Social Robotics, vol. 6, no. 2, 2014, pp. 299310.

76. R. Kachouie, S. Sedighadeli, R. Khosla, and M.T. Chu, 'Socially assistive robots in elderly care: a mixed-method systematic literature review', International Journal of Human-Computer Interaction, vol. 30, no. 5, 2014, pp. 369-393.

77. H. Osawa, J. Orszulak, K.M. Godfrey, and J.F. Coughlin, 'Maintaining learning motivation of older people by combining household appliance with a communication robot', In: Proceedings of the 2010 IEEE/RSJ International Conference on Intelligent Robots and Systems, 2010, pp. 5310- 5316.

78. J. Forlizzi, C. DiSalvo, and F. Gemperle, 'Assistive robotics and an ecology of elders living independently in their homes', Human-Computer Interaction, vol. 19, no. 1, 2004, pp. 25-59.

79. J.G. Anderson, 'Social, ethical and legal barriers to ehealth', International Journal of Medical Informatics, vol. 76, no. 5, 2007, pp. 480-483. 